PROCEEDINGS OF THE

AMERICAN MATHEMATICAL SOCIETY

Volume 127, Number 6, Pages 1863-1871

S 0002-9939(99)04757-7

Article electronically published on February 17, 1999

\title{
FUNCTION ALGEBRAS AND THE LATTICE OF COMPACTIFICATIONS
}

\author{
FRANKLIN MENDIVIL
}

(Communicated by Alan Dow)

\begin{abstract}
We provide some conditions as to when $K(X) \cong K(Y)$ for two locally compact spaces $X$ and $Y$ (where $K(X)$ is the lattice of all Hausdorff compactifications of $X)$. More specifically, we prove that $K(X) \cong K(Y)$ if and only if $C^{*}(X) / C_{0}(X) \cong C^{*}(Y) / C_{0}(Y)$. Using this result, we prove several extensions to the case where $K(X)$ is embedded as a sub-lattice of $K(Y)$ and to where $X$ and $Y$ are not locally compact.

One major contribution is in the use of function algebra techniques. The use of these techniques makes the extensions simple and clean and brings new tools to the subject.
\end{abstract}

\section{INTRODUCTION}

The study of the Hausdorff compactification of a Tychonoff space $X$ is a wellestablished branch of topology (the books $[\mathrm{PW}]$ and $[\mathrm{Ch}]$ are nice references in this area). There are many results examining the types of compactifications that $X$ may have or the structure of the collection of all the compactifications of $X$. In fact, if we denote by $K(X)$ the collection of all Hausdorff compactifications of $X$, we may put an order structure on $K(X)$ which makes it a complete upper semi-lattice (which is a lattice if and only if $X$ is locally compact).

In this paper, we examine when $K(X)$ is isomorphic to $K(Y)$ for two spaces $X$ and $Y$. We also examine when $K(X)$ can be embedded in $K(Y)$.

Under what conditions are $K(X)$ and $K(Y)$ isomorphic? One simple sufficient condition is as follows. Suppose that $X_{0}$ and $Y_{0}$ are compact with $X_{0} \subset X$ and $Y_{0} \subset Y$. If $X \backslash X_{0}$ is homeomorphic to $Y \backslash Y_{0}$, then $K(X)$ is isomorphic to $K(Y)$.

This is by no means the whole story, however. Let $W(\alpha)=\{\gamma \mid \gamma<\alpha\}$ for an ordinal $\alpha$ and put the order topology on $W(\alpha)$. Let $\omega_{\alpha}$ be the smallest ordinal of cardinality $\aleph_{\alpha}$. Then $K\left(W\left(\omega_{\alpha}\right)\right)$ is trivial if $\alpha \geq 1$, (4.13 of [Ch]). Thus, $K\left(W\left(\omega_{\alpha}\right)\right) \cong K\left(W\left(\omega_{\delta}\right)\right)$ if $\alpha, \delta \geq 1$. Since $\omega_{\alpha}$ and $\omega_{\delta}$ have different cardinalities, $W\left(\omega_{\alpha}\right)$ and $W\left(\omega_{\delta}\right)$ are not homeomorphic. In fact, if $C \subset W\left(\omega_{\alpha}\right)$ is any compact set, then $W\left(\omega_{\alpha}\right) \backslash C$ has the same cardinality as $W\left(\omega_{\alpha}\right)$. Thus, $W\left(\omega_{\alpha}\right) \backslash C$ and $W\left(\omega_{\delta}\right) \backslash D$ cannot be homeomorphic for any compact sets $C$ and $D$. This example shows that our simple sufficient condition is not necessary in general.

Received by the editors December 10, 1996 and, in revised form, September 22, 1997.

1991 Mathematics Subject Classification. Primary 54D35, 54C35, 54D40, 46E25.

Key words and phrases. Function algebras, compactifications, lattice of compactifications, maximal ideals, structure space, rings of continuous functions. 
Thus, we need to look for other conditions; Magill provided one such condition in his paper [MG2]. Theorem 12 and Theorem 13 from this paper yield the following result:

Theorem 1 (Magill). Let $X$ and $Y$ be two locally compact Tychonoff spaces. Then $K(X)$ is isomorphic to $K(Y)$ if and only if $\beta X \backslash X$ is homeomorphic to $\beta Y \backslash Y$.

In this paper, we prove a theorem (Theorem 12) which implies Magill's result. Our use of function algebra techniques brings new tools to this area. We also prove several extensions of Magill's result, most of them with simple proofs using the new techniques. Using algebraic techniques, many of the necessary manipulations can be handled naturally by the algebra.

All spaces will be Tychonoff. The main results are Theorems 12 and 15. The other theorems are basically refinements of these results.

\section{Preliminaries}

Notational conventions. If $\mathcal{I}$ and $\mathcal{J}$ are two (closed) subalgebras of a function algebra $\mathcal{A}$, we denote by $\mathcal{I} \oplus \mathcal{J}$ the (closed) subalgebra of $\mathcal{A}$ generated by $\mathcal{I}$ and $\mathcal{J}$. As a special case, we denote by $\mathcal{I} \oplus 1$ the algebra generated by $\mathcal{I}$ and the constant functions.

Since the algebraic sum of $\mathcal{I}$ and the constant functions is closed if and only if $\mathcal{I}$ is closed, taking the closure is unnecessary in this case.

Further, if $\left\{\mathcal{I}_{\alpha}\right\}$ is a collection of elements in some partially ordered set $\mathcal{P}$, we denote by $\bigvee\left\{\mathcal{I}_{\alpha}\right\}$ the smallest element of $\mathcal{P}$ larger than all of the $\mathcal{I}_{\alpha}$ 's, when it exists. Similarly, we denote by $\bigwedge\left\{\mathcal{I}_{\alpha}\right\}$ the largest element of $\mathcal{P}$ smaller than all of the $\mathcal{I}_{\alpha}$ 's, when it exists.

For a collection of functions $\mathcal{F}$ on $X, H \subset X$ is a stationary set of $\mathcal{F}$ if $\left.f\right|_{H}$ is constant for each $f \in \mathcal{F}$. We will usually be interested in the maximal stationary sets. We denote by $\mathbf{Z}(\mathcal{I})=\{x \mid f(x)=0, \forall f \in \mathcal{I}\}$, which we call the zero-set of the ideal $\mathcal{I}$ (even though it may not be a zero set as defined in [GJ]).

The following proposition is well known, but we include it for completeness.

Proposition 2. Let $X$ be a compact Hausdorff space and $\mathcal{I} \subset C^{*}(X)$ be a closed ideal. Then $\mathcal{I}=\left\{f:\left.f\right|_{\mathbf{z}(\mathcal{I})}=0\right\}$.

Proof. Let $S$ be a nonsingleton stationary set of $\mathcal{I}$. Suppose that $f \in \mathcal{I}$ and $\left.f\right|_{S} \neq 0$. Let $g \in C^{*}(X)$ be such that $\left.g\right|_{S}$ is not constant. Then $f g \in \mathcal{I}$ and is not constant on $S$. Thus, the only value any $f \in \mathcal{I}$ can take on $S$ is 0 .

By the Stone-Weierstrass Theorem (16.3 in [GJ]), I $\oplus 1$ is the set of all functions constant on the stationary sets of $\mathcal{I}$.

If $\alpha X \leq \gamma X \in K(X)$, then we denote by $[\alpha X, \gamma X]$ the order interval of all compactifications between $\alpha X$ and $\gamma X$, inclusive.

Now we will state a couple of easy results which will prove useful to us in the sequel. The second one is just the Chinese Remainder Theorem for $C^{*}(H)$ for compact Hausdorff $H$. We leave the proofs to the interested reader.

Proposition 3. Let $H$ be a compact Hausdorff space and let $\mathcal{I} \subset C^{*}(H)$ be a closed ideal. Let $S=\mathbf{Z}(\mathcal{I})$, then $C^{*}(H) / \mathcal{I} \cong C^{*}(S)$.

Lemma 4 (Chinese Remainder Theorem). Let $\mathcal{I}_{i}$ be ideals in $C^{*}(H)$ for $i=1, \ldots$, n. Suppose that $\mathcal{I}_{i} \bigvee \mathcal{I}_{j}=C^{*}(H)$ for all $i \neq j$. Let $\mathcal{I}=\bigcap \mathcal{I}_{i}$. Then $C^{*}(H) / \mathcal{I} \cong$ $C^{*}(H) / \mathcal{I}_{1} \oplus \cdots \oplus C^{*}(H) / \mathcal{I}_{n}$. 
To prove Lemma 4, one need only use the usual Chinese Remainder Theorem to get the algebraic isomorphism and then prove it to be an isomorphism of $C^{*}$ algebras.

Lemma 5. Let $X$ be locally compact. Then $C^{*}(X) / C_{0}(X) \cong C^{*}(\beta X \backslash X)$.

Proof. $C_{0}(X)$ is an ideal in $C^{*}(X)$ and $C_{0}(X)=\left\{f \in C^{*}(X)\left|f^{\beta}\right|_{\beta X \backslash X}=0\right\}$ where $f^{\beta}$ is the extension of $f$ to $\beta X$. Now the lemma follows from Proposition 3 using $\mathcal{I}=C_{0}(X)$ and $H=\beta X$.

Definition 1. $P S A(X)=$ lattice of all closed unital subalgebras of $C^{*}(X)$ which separate points from closed sets.

$C A(X)=$ lattice of all closed unital subalgebras of $C^{*}(X)$.

$N I(X)=$ the partially ordered set of all closed non-maximal ideals in $C^{*}(X)$.

$M I(X)=$ the collection of all maximal ideals in $C^{*}(X)$.

Lemma 6. Let $X$ be locally compact. Then $P S A(X) \cong C A(\beta X \backslash X)$.

Proof. Define $\Upsilon: C^{*}(X) \rightarrow C^{*}(\beta X \backslash X)$ by $\Upsilon(f)=\left.f^{\beta}\right|_{\beta X \backslash X} ; \Upsilon$ is the homomorphism that induces the isomorphism in Lemma 5. We define a function $\Upsilon^{\#}: P S A(X) \rightarrow C A(\beta X \backslash X)$ by $\mathcal{A} \mapsto\{\Upsilon(f): f \in \mathcal{A}\}$. Now, from the correspondence theorem $\left([\mathrm{RO}]\right.$, p. 26) from elementary algebra, $\Upsilon^{\#}$ is an order preserving bijection between the set of subalgebras of $C^{*}(X)$ which contain $\operatorname{ker}(\Upsilon)=C_{0}(X)$ and the subalgebras of $C^{*}(\beta X \backslash X)$. However, for a locally compact space, an algebra $\mathcal{A} \in C A(X)$ is in $P S A(X)$ if and only if $C_{0}(X) \subset \mathcal{A}$.

There is a nice discussion of compactifications from the point of view of upper semicontinuous (u.s.c.) decompositions in the papers by Firby $[F]$. Each compactification of a locally compact space $X$ induces an u.s.c. decomposition on $\beta X \backslash X$. In fact, the set of u.s.c. decompositions of $\beta X \backslash X$ is isomorphic to the lattice of compactifications of a locally compact space (see section 1.1 in $[\mathrm{F}]$ ).

A u.s.c. decomposition is called primary if it has only one nonsingleton block.

Given subalgebra $\mathcal{A} \subset C^{*}(X), \mathcal{A}$ induces a u.s.c. decomposition on $\beta X \backslash X$ via the stationary sets of the extension of $\mathcal{A}$ to $\beta X$. Thus, we also get a correspondence between the u.s.c. decompositions of $\beta X \backslash X$ and closed unital algebras.

By Theorem 4.5 (q) in $[\mathrm{PW}]$, we know that $P S A(X) \cong K(X)$ for a Tychonoff space $X$. For the special case of locally compact Hausdorff spaces, another way of stating this is that there is a lattice isomorphism between the lattice of all those algebras in $C A(X)$ which contain $C_{0}(X)$ and $K(X)$. This is since for a locally compact space $X$, an algebra $\mathcal{A} \in C A(X)$ separates points from closed sets if and only if $C_{0}(X) \subset \mathcal{A}$.

We are particularly interested in closed unital algebras $\mathcal{A}$ of the form $\mathcal{A}=\mathcal{I} \oplus 1$ for some closed ideal $\mathcal{I}$. The following proposition characterizes these in $C A(X)$.

In the lattice $C A(X)$, a dual point is some $\mathcal{A} \in C A(X)$ with $\mathcal{A}<C^{*}(X)$ and for all $\mathcal{B} \in C A(X)$ with $\mathcal{A} \leq \mathcal{B}$ either $\mathcal{B}=C^{*}(X)$ or $\mathcal{A}=\mathcal{B}$. In terms of u.s.c. decompositions, a dual point $\mathcal{A}$ is an algebra whose corresponding u.s.c. decomposition is primary and whose nonsingleton block contains exactly two points.

Proposition 7. Let $X$ be a Tychonoff space and $\mathcal{A} \in C A(X)$. Then $\mathcal{A}=\mathcal{I} \oplus 1$ for some closed ideal $\mathcal{I}$ if and only if $\left[\mathcal{A}, C^{*}(X)\right] \cong C A(S)$ for some compact Hausdorff space $S$. 
Proof. We can assume that $X$ is compact, since if it is not we simply replace $X$ with $\beta X$. Since $C^{*}(X) \cong C^{*}(\beta X)$, this does not affect any of the algebraic or order relations.

First suppose that $\mathcal{A}=\mathcal{I} \oplus 1$, then by Propositions 2 and $3, C^{*}(X) / \mathcal{I} \cong C^{*}(S)$ where $S=Z(\mathcal{I})$. Now this isomorphism takes $\mathcal{B} \in\left[\mathcal{A}, C^{*}(X)\right]$ to some closed unital algebra in $C A(S)$. Furthermore, this correspondence between elements in $\left[\mathcal{A}, C^{*}(X)\right]$ and closed unital algebras in $C^{*}(S)$ is order preserving and bijective. Thus, $C A(S) \cong\left[\mathcal{A}, C^{*}(X)\right]$.

Conversely, suppose that $\left[\mathcal{A}, C^{*}(X)\right] \cong C A(S)$ for some compact Hausdorff $S$. Denote this isomorphism by $\Sigma$. We wish to show that $\mathcal{A}=\mathcal{I} \oplus 1$ for some ideal $\mathcal{I}$. Suppose not. Then there are two non-maximal ideals $\mathcal{I}_{1}$ and $\mathcal{I}_{2}$ so that $\left(\mathcal{I}_{1} \oplus 1\right) \bigvee\left(\mathcal{I}_{2} \oplus 1\right)=C^{*}(X)$ and $\mathcal{A} \leq\left(\mathcal{I}_{1} \oplus 1\right) \wedge\left(\mathcal{I}_{2} \oplus 1\right)$. Let $C_{1}$ and $C_{2}$ be dual points in $C A(X)$ with $\mathcal{I}_{1} \oplus 1 \leq C_{1}$ and $\mathcal{I}_{2} \oplus 1 \leq C_{2}$. Then $C_{1}$ and $C_{2}$ are the only dual points larger than $C_{1} \wedge C_{2}$.

Because of the special structure of $C A(S)$, there is some dual point $D_{3} \in C A(S)$ so that there are exactly six dual points larger than $\Sigma\left(C_{1}\right) \wedge \Sigma\left(C_{2}\right) \wedge D_{3}$. Thus, there are exactly six dual points larger than $C_{1} \wedge C_{2} \wedge \Sigma^{-1}\left(D_{3}\right)$ (since $\Sigma$ is an isomorphism). However, since $C_{1} \wedge C_{2}=\mathcal{A} \wedge C_{1} \wedge C_{2} \leq \Sigma^{-1}\left(D_{3}\right) \wedge C_{1} \wedge C_{2} \leq$ $C_{1} \wedge C_{2}$, this contradicts the choice of $C_{1}$ and $C_{2}$. Thus, no such $C_{1}$ and $C_{2}$ exist so no such $\mathcal{I}_{1}$ and $\mathcal{I}_{2}$ exist.

We note that if $\mathcal{A}=\mathcal{I} \oplus 1$, then the u.s.c decomposition induced by $\mathcal{A}$ is primary (this is essentially the content of Proposition 2).

\section{MAIN RESUlTS}

The main result of this section is Theorem 12, which gives an algebraic condition on $C^{*}(X)$ and $C^{*}(Y)$ for when $K(X)$ is isomorphic to $K(Y)$. Using this, we then get some results as to when an interval in $K(X)$ can be embedded as an interval of $K(Y)$.

Now we prove a series of results with the goal of proving Theorem 12. The propositions show the major steps in the proof of this theorem. Theorem 11 is of independent interest since it indicates that the lattice structure of $C A(X)$ is sufficient to determine $C^{*}(X)$.

Proposition 8. Let $X$ and $Y$ be compact Hausdorff spaces. Suppose that there is a lattice isomorphism $\phi: C A(X) \rightarrow C A(Y)$. Then there is an isomorphism of partially ordered sets $\psi: N I(X) \rightarrow N I(Y)$.

Proof. We now define $\psi$ by using this characterization of algebras of $\mathcal{A}$ of the form $\mathcal{A}=\mathcal{I} \oplus 1$ obtained in Proposition 7. Given $\mathcal{A}=\mathcal{I} \oplus 1$, define $\psi(\mathcal{I})=\mathcal{J}$ where $\phi(\mathcal{I} \oplus 1)=\mathcal{J} \oplus 1$. This is well-defined since $\mathcal{J}$ is unique because $\mathcal{I}$ is non-maximal. Clearly $\psi$ is bijective and order preserving.

Proposition 9. Let $X$ and $Y$ be compact Hausdorff spaces. An isomorphism of partially ordered sets $\psi: N I(X) \rightarrow N I(Y)$ induces a bijection $F_{\psi}: M I(X) \rightarrow$ $M I(Y)$.

Proof. Let $M_{x} \in M I(X)$, and let $\left\{\mathcal{I}_{\alpha}\right\}$ be an infinite family in $N I(X)$ which is directed upward by inclusion, so that $M_{x}=\bigvee_{\alpha} \mathcal{I}_{\alpha}$. Define $F_{\psi}\left(M_{x}\right)=\bigvee_{\alpha} \psi\left(\mathcal{I}_{\alpha}\right)$. First we show that this is a maximal ideal, then we prove that it is well-defined. Clearly $N=F_{\psi}\left(M_{x}\right) \notin N I(Y)$ (else $\mathcal{I}_{\alpha} \leq \psi^{-1}\left(F_{\psi}\left(M_{x}\right)\right) \in N I(X)$ for all $\alpha$ ). 
Thus suppose that $N=C^{*}(Y)$. This implies that $\bigcap_{\lambda} Z\left(\psi\left(\mathcal{I}_{\lambda}\right)\right)=\emptyset$. However, since $\mathcal{I}_{\lambda_{1}} \leq \mathcal{I}_{\lambda_{2}}$ for $\lambda_{1} \leq \lambda_{2}$ then $\psi\left(\mathcal{I}_{\lambda_{1}}\right) \leq \psi\left(\mathcal{I}_{\lambda_{2}}\right)$, so $Z\left(\psi\left(\mathcal{I}_{\lambda_{1}}\right)\right) \supset Z\left(\psi\left(\mathcal{I}_{\lambda_{2}}\right)\right)$ is a directed collection of compact sets with the finite intersection property but with empty intersection. Thus there is an $\lambda_{0} \in \lambda$ so that $\mathcal{Z}\left(\psi\left(\mathcal{I}_{\lambda_{0}}\right)=\emptyset\right.$, which implies that $Z\left(\psi\left(\mathcal{I}_{\lambda}\right)\right)=\emptyset$ and this contradicts $\psi\left(\mathcal{I}_{\lambda}\right) \in N I(Y)$. Thus $N \neq C^{*}(Y)$ so $N$ is a maximal ideal in $C^{*}(Y)$.

To prove that $F_{\psi}$ is well-defined, suppose $\left\{\mathcal{I}_{\alpha}\right\}_{\alpha \in \Lambda}$ and $\left\{\mathcal{J}_{\gamma}\right\}_{\gamma \in \Xi}$ are two such nets with $\bigvee_{\alpha} \mathcal{I}_{\alpha}=M_{x}=\bigvee_{\gamma} \mathcal{J}_{\gamma}$. Let $K_{(\alpha, \gamma)}=\mathcal{I}_{\alpha} \wedge \mathcal{J}_{\gamma}$. Then $\bigvee_{\omega} K_{\omega}=M_{x}$. Let $N=\bigvee_{\alpha} \psi\left(\mathcal{I}_{\alpha}\right)$ and $N^{\prime}=\bigvee_{\gamma} \psi\left(\mathcal{J}_{\gamma}\right)$ and $N^{\prime \prime}=\bigvee_{\omega} \psi\left(K_{\omega}\right)$. Then $N^{\prime \prime} \leq N$ and $N^{\prime \prime} \leq N^{\prime}$ so $N=N^{\prime \prime}=N^{\prime}$ since they are all maximal ideals.

Since $F_{\psi^{-1}}$ is an inverse for $F_{\psi}$, we know that $F_{\psi}$ is bijective.

Proposition 10. Let $X$ and $Y$ be compact Hausdorff spaces and $\psi: N I(X) \rightarrow$ $N I(Y)$ be an isomorphism of partially ordered sets. The function $F_{\psi}$ is a homeomorphism when we put the hull-kernel topology on both $M I(X)$ and $M I(Y)$.

Proof. In Proposition 9 we have shown that $F_{\psi}$ is a bijection. Thus all that remains is to show that $F_{\psi}$ is continuous, which we do by proving that $F_{\psi}(\operatorname{cl}(A)) \subset$ $\operatorname{cl}\left(F_{\psi}(A)\right)$.

The closure of $A \subset M I(X)$ in the hull-kernel topology is the set

$$
c l(A)=\{\mathcal{M} \in M I(X) \mid \mathcal{M} \supset \bigcap\{I \mid I \in A\}\} .
$$

Thus

$$
F_{\psi}(c l(A))=\left\{F_{\psi}(M) \mid M \supset \bigcap\{I \mid I \in A\}\right\}
$$

and

$$
\operatorname{cl}\left(F_{\psi}(A)\right)=\left\{N \in M I(Y) \mid N \supset \bigcap\left\{F_{\psi}(I) \mid I \in A\right\}\right\} .
$$

Let $\mathcal{I}=\bigcap\{I \mid I \in A\} \in N I(X)$ and $\mathcal{J}=\bigcap\left\{F_{\psi}(I) \mid I \in A\right\} \in N I(Y)$. Take $M \in M I(X)$ so that $\mathcal{I} \subset M$. We wish to show that $F_{\psi}(M) \supset \mathcal{J}$. Now $\mathcal{J} \subset F_{\psi}(I)$ for all $I \in A$ so $\psi^{-1}(\mathcal{J}) \subset F_{\psi^{-1}}\left(F_{\psi}(I)\right)=I$ for all $I \in A$. Thus $\psi^{-1}(\mathcal{J}) \subset \mathcal{I}$ so $\mathcal{J} \subset \psi(\mathcal{I})$. However, $\psi(\mathcal{I}) \subset F_{\psi}(M)$ so $\mathcal{J} \subset \psi(\mathcal{I}) \subset F_{\psi}(M)$. Thus if $M \in \operatorname{cl}(A)$ then $F_{\psi}(M) \in \operatorname{cl}\left(F_{\psi}(A)\right)-$ so $F_{\psi}$ is continuous.

By symmetry, $F_{\psi^{-1}}=F_{\psi}^{-1}$ is continuous. Thus, $F_{\psi}$ is a homeomorphism.

Theorem 11. Let $X$ and $Y$ be compact Hausdorff spaces. Then $C A(X)$ is lattice isomorphic to $C A(Y)$ if and only if $C^{*}(X)$ is isomorphic to $C^{*}(Y)$.

Proof. From Propositions 8, 9, and 10 we know if $C A(X)$ is isomorphic to $C A(Y)$, then $M I(X)$ is homeomorphic to $M I(Y)$. However, it is well know that $M I(X)$ with the hull-kernel topology is homeomorphic to $X$. Thus $X$ and $Y$ are homeomorphic. However, then clearly $C^{*}(X)$ and $C^{*}(Y)$ are isomorphic.

The proof of the converse is straightforward.

Theorem 12. Let $X$ and $Y$ be locally compact spaces. Then $K(X) \cong K(Y)$ if and only if $C^{*}(X) / C_{0}(X) \cong C^{*}(Y) / C_{0}(Y)$.

Proof. One direction is easy, the other much harder. First, suppose $C^{*}(X) / C_{0}(X)$ $\cong C^{*}(Y) / C_{0}(Y)$. Then by Lemma 5 , we know $C^{*}(\beta X \backslash X) \cong C^{*}(\beta Y \backslash Y)$, so that $C A(\beta X \backslash X) \cong C A(\beta Y \backslash Y)$. However, then by Lemma $6, P S A(X) \cong P S A(Y)$, but $P S A(X) \cong K(X)$ and similarly for $\mathrm{Y}$. Thus, $K(X) \cong K(Y)$. 
Conversely, suppose $K(X) \cong K(Y)$. Then, as before, $P S A(X) \cong P S A(Y)$ so, by Lemma $6, C A(\beta X \backslash X) \cong C A(\beta Y \backslash Y)$. Now Theorem 11 then gives us that $C^{*}(\beta X \backslash X)$ is isomorphic to $C^{*}(\beta Y \backslash Y)$. However, then $C^{*}(X) / C_{0}(X)$ is isomorphic to $C^{*}(Y) / C_{0}(Y)$.

The following diagram will help in illustrating the spaces and isomorphism involved.

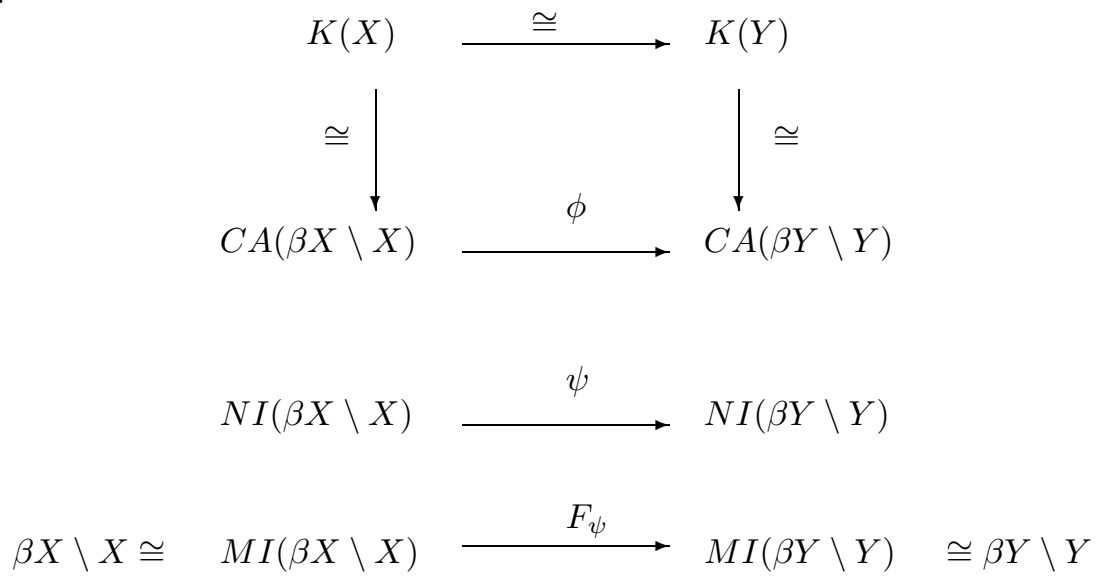

DiAgRAM FOR THEOREM 12

Remark. It is well known that if $S$ and $T$ are compact Hausdorff spaces, then $S$ is homeomorphic to $T$ if and only if $C^{*}(S) \cong C^{*}(T)$ [GJ]. This fact and Lemma 5 show that Theorem 12 implies Magill's Theorem.

Let $\mathcal{C}$ be a closed unital subalgebra. Then there are many non-unital closed subalgebras $\mathcal{A}$ so that $\mathcal{C}=\mathcal{A} \oplus 1$ (in fact any such $\mathcal{A}$ will be a maximal ideal in $\mathcal{C})$. Now, for each compactification $\alpha X$, there corresponds a unique closed unital subalgebra $C_{\alpha}$. However, then there will be many closed non-unital subalgebras corresponding to $\alpha X$. Thus, the lattice of all closed subalgebras of $C^{*}(X)$ is much larger than the lattice of all compactifications of $X$. This is why we need to assume that the algebras are unital in Theorem 12.

We can generalize Theorem 12 some to the situation where we have a sub-lattice of $K(X)$ lattice isomorphic to a sub-lattice of $K(Y)$. Here, the two sub-lattices must be intervals, and the lower bound of each interval must satisfy some additional hypothesis.

We recall the definition of $C_{\alpha}(X)$.

Definition 2. For $\alpha X \in K(X)$, we define $C_{\alpha}(X)=\left\{f \in C^{*}(X) \mid f\right.$ extends to $\alpha X\}$.

Now we record a fact which we need in the proofs of several of the succeeding results. The following lemma is a variation and slight generalization of Lemma 2.2.1 from $[\mathrm{F}]$.

Lemma 13. Let $X$ be a space and $\alpha X \leq \delta X \in K(X)$. Then $[\alpha X, \delta X]$ is lattice isomorphic to the collection of all closed unital subalgebras of $C_{\delta}$ which contain $C_{\alpha}$. If $C_{\alpha}=\mathcal{I} \oplus 1$ for some ideal $\mathcal{I}$, then this is isomorphic to the lattice of all closed unital algebras of $C_{\delta} / \mathcal{I}$. 
Proof. The isomorphism between $K(X)$ and $P S A(X)$ carries the interval $[\alpha X, \delta X]$ bijectively onto the set of all closed algebras between $C_{\alpha}$ and $C_{\delta}$.

Once we note this, the proof of the second remark is similar to the proof of Lemma 6.

We never explicitly used the fact that $X$ was locally compact in the proofs of Lemmas 5 and 6 . The only place it was used is in the fact that $C_{0}(X)$ separates points from closed sets. We simply replace $C_{0}(X)$ by $\mathcal{I}$, and the same proofs go through when $X$ is not locally compact.

In the language of u.s.c. decompositions, the second conclusion in the lemma simply states that the lattice of u.s.c. decompositions of $\delta X \backslash X$ above the primary u.s.c. decomposition generated by $\alpha X$ is isomorphic to the lattice of u.s.c. decompositions of $\mathbf{Z}(\mathcal{I})$ considered as a subset of $\delta X \backslash X$.

Theorem 14. Let $X$ and $Y$ be spaces, $\alpha X \leq \delta X \in K(X)$ and $\gamma Y \leq \theta Y \in K(Y)$. Suppose that $\alpha X$ and $\gamma Y$ are such that $C_{\alpha}=\mathcal{I} \oplus 1$ and $C_{\gamma}=\mathcal{J} \oplus 1$ for some ideals $\mathcal{I} \subset C_{\delta}$ and $\mathcal{J} \subset C_{\theta}$. Then $[\alpha X, \delta X] \cong[\gamma Y, \theta Y]$ if and only if $C_{\delta}(X) / \mathcal{I} \cong$ $C_{\theta}(Y) / \mathcal{J}$.

Proof. $C_{\delta} / \mathcal{I} \cong C^{*}(S)$ with $S=\mathbf{Z}(\mathcal{I})$. Similarly, $C_{\theta} / \mathcal{J} \cong C^{*}(T)$ with $T=\mathbf{Z}(\mathcal{J})$. Using this observation, the proof follows from Theorem 11 in a similar manner as Theorem 12 .

Denoting by $\omega X$ the one-point compactification of $X$, then $C_{\omega}=C_{0}(X) \oplus 1$. Thus, the one-point compactification satisfies the additional hypothesis that are needed in the generalization of Theorem 12. Further, clearly $\omega X \leq \beta X$. Thus Theorem 12 is a special case of Theorem 14 .

Let $\delta X=\beta X, \theta Y=\beta Y$, and $\alpha X$ and $\gamma Y$ be primary compactifications (i.e., compactifications whose corresponding u.s.c. decompositions are primary) so that $C_{\alpha}=\mathcal{I} \oplus 1$ and $C_{\gamma}=\mathcal{J} \oplus 1$ for some ideals $\mathcal{I}$ and $\mathcal{J}$. Denote by $B_{\alpha}$ and $B_{\gamma}$ the nonsingleton block for $\alpha X$ and $\gamma Y$ respectively. Theorem 14 states that $[\alpha X, \beta X] \cong[\gamma Y, \beta Y]$ if and only if $B_{\alpha} \cong B_{\gamma}$ and thus is an extension of Magill's Theorem (Theorem 1).

We can obtain another slight generalization to the case where the lower bounds of the intervals are not of the form $C_{\alpha}=\mathcal{I} \oplus 1$. However, we do need some hypotheses on the lower bounds.

Theorem 15. Let $X$ and $Y$ be spaces.

Let $\alpha_{i} X \in K(X)$ for $i=1, \ldots, n$ with $C_{\alpha_{i}}=\mathcal{I}_{i} \oplus 1$ for some ideals $\mathcal{I}_{i} \subset C_{\delta}$ with $\mathcal{I}_{i} \bigvee \mathcal{I}_{j}=C_{\delta}$ if $i \neq j$. Let $\mathcal{I}=\bigcap \mathcal{I}_{i}$ and $\alpha X \leq \delta X \in K(X)$ with $\alpha X=\bigwedge \alpha_{i} X$.

If $[\alpha X, \delta X] \cong[\gamma Y, \theta Y]$ for some $\gamma Y \leq \theta Y \in K(Y)$, then there are $\gamma_{i} Y \in K(Y)$ so that $\gamma Y=\bigwedge \gamma_{i} Y$ with $C_{\gamma_{i}}=\mathcal{J}_{i} \oplus 1$ for some ideals $\mathcal{J}_{i} \subset C_{\theta}$. Furthermore, letting $\mathcal{J}=\bigcap \mathcal{J}_{i}$, we have $C_{\delta} / \mathcal{I} \cong C_{\theta} / \mathcal{J}$.

Conversely, if there are compactifications $\gamma^{\prime} Y \leq \theta Y \in K(Y)$ with $C_{\gamma^{\prime}}=\mathcal{J} \oplus 1$ for some ideal $\mathcal{J} \subset C_{\theta}$ and such that $C_{\theta} / \mathcal{J} \cong C_{\delta} / \mathcal{I}$ then $[\alpha X, \delta X] \cong[\gamma Y, \theta Y]$ for some $\gamma Y \geq \gamma^{\prime} Y$. Furthermore, there are compactifications $\gamma_{i} Y \in K(Y)$ so that $C_{\gamma_{i}}=\mathcal{J}_{i} \oplus 1$ for some ideals $\mathcal{J}_{i} \subset C_{\theta}$ and $\mathcal{J}=\bigcap \mathcal{J}_{i}$ and $\mathcal{J}_{i} \bigvee \mathcal{J}_{j}=C_{\theta}$ for $i \neq j$ and $\gamma Y=\bigwedge \gamma_{i} Y$.

Proof. First, suppose that $[\alpha X, \delta X] \cong[\gamma Y, \theta Y]$ and let us denote this isomorphism by $\Gamma$. Then clearly there are $\gamma_{i} Y \in[\gamma Y, \theta Y]$ so that $\gamma Y=\bigwedge \gamma_{i} Y$ and $\Gamma\left(\alpha_{i} X\right)=\gamma_{i} Y$ (since $\Gamma$ is an isomorphism). Furthermore by Proposition 8 we know that $C_{\gamma_{i}}=$ 
$\mathcal{J}_{i} \oplus 1$ for some ideals $\mathcal{J}_{i} \subset C_{\theta}$ since $C_{\alpha_{i}}=\mathcal{I}_{i} \oplus 1$. Thus, the only thing left to show is that $C_{\delta} / \mathcal{I} \cong C_{\theta} / \mathcal{J}$. Since $\alpha X \leq \alpha_{i} X \leq \delta X$, we know that $\left[\alpha_{i} X, \delta X\right]$ is a sublattice of $[\alpha X, \delta X]$ so that $\left[\alpha_{i} X, \delta X\right] \cong\left[\gamma_{i} Y, \theta Y\right]$ under $\Gamma$. Therefore, by Theorem 14, $C_{\delta} / \mathcal{I}_{i} \cong C_{\theta} / \mathcal{J}_{i}$ for $i=1, \ldots, n$. However, then by Lemma 4 (Chinese Remainder Theorem), $C_{\delta} / \mathcal{I} \cong C_{\delta} / \mathcal{I}_{1} \oplus \cdots \oplus C_{\delta} / \mathcal{I}_{n}$ and similarly for $C_{\theta} / \mathcal{J}$ (this is since $\mathcal{I}_{i} \bigvee \mathcal{I}_{j}=C_{\delta}$ if $i \neq j$ ). Thus, $C_{\delta} / \mathcal{I} \cong C_{\theta} / \mathcal{J}$.

Conversely, suppose that $C_{\delta} / \mathcal{I} \cong C_{\theta} / \mathcal{J}$. Let $\alpha^{\prime} X \in K(X)$ such that $C_{\alpha^{\prime}}=\mathcal{I} \oplus 1$ and, similarly, let $\gamma^{\prime} Y \in K(Y)$ so that $C_{\gamma^{\prime}}=\mathcal{J} \oplus 1$ (this is possible since $\mathcal{I}$ separates points from closed sets in $X$ and $\mathcal{J}$ separates points from closed sets in $Y$ ). Then, by Theorem 14, $\left[\alpha^{\prime} X, \delta X\right] \cong\left[\gamma^{\prime} Y, \theta Y\right]$. However, $\alpha^{\prime} X \leq \alpha X$ since $\mathcal{I} \subset \mathcal{I}_{i}$ and, similarly, $\gamma^{\prime} Y \leq \gamma Y$. Thus, $[\alpha X, \delta X]$ is a sublattice of $\left[\alpha^{\prime} X, \gamma X\right]$ and $[\gamma Y, \theta Y]$ is a sublattice of $\left[\gamma^{\prime} Y, \theta Y\right]$ and the isomorphism between $\left[\alpha^{\prime} X, \delta X\right]$ and $\left[\gamma^{\prime} Y, \theta Y\right]$ takes $[\alpha X, \delta X]$ to $[\gamma Y, \theta Y]$. Thus, $[\alpha X, \delta X] \cong[\gamma Y, \theta Y]$.

Very roughly, Theorem 15 says that if you have an isomorphism between $[\alpha X, \delta X]$ and $[\gamma Y, \theta Y]$ where $\alpha X$ is the meet of finitely many compactifications whose corresponding u.s.c. decompositions are primary, then you can extend this isomorphism all the way down to the compactification whose corresponding u.s.c. decomposition is primary and has as nonsingleton block the union of the blocks of the $\alpha_{i} X$ 's.

We could extend this theorem to infinitely many $\alpha_{i} X$ 's under the assumption that if $C_{\delta} / \mathcal{I}_{i} \cong C_{\theta} / \mathcal{J}_{i}$ for all $i$ in some indexing set $\Lambda$, then $C_{\delta} / \mathcal{I} \cong C_{\theta} / \mathcal{J}$ where $\mathcal{I}=\bigcap \mathcal{I}_{i}$ and $\mathcal{J}=\bigcap \mathcal{J}_{i}$. However, this assumption is not true in general if there are infinitely many $\mathcal{I}_{i}$ 's. Here is an example of this.

Example. Let $Y=X=\mathbb{R}$ and $\delta X=\theta Y$ be such that $\delta X \backslash X=[0,1]$ (we know that such compactifications exist, e.g. see $[\mathrm{AB}])$. Let $S_{i}=\{1 / i\} \subset[0,1]$ for $i=1,2,3,4, \ldots$ and $S_{0}=\{0\}$. Let $T_{2 i}=\{1 / i\}$ and $T_{2 i+1}=\{1-1 / i\}$ for $i=1,2,3,4, \ldots$, and $T_{0}=\{0\}$ and $T_{1}=\{1\}$. Now, let $\mathcal{I}_{i}=\left\{f \in C_{\delta}|f|_{S_{i}}=0\right\}$ and $\mathcal{J}_{i}=\left\{f \in C_{\theta}|f|_{T_{i}}=0\right\}$. Finally, let $\mathcal{I}=\bigcap \mathcal{I}_{i}$ and $\mathcal{J}=\bigcap \mathcal{J}_{i}$. Then clearly $C_{\delta} / \mathcal{I}_{i} \cong C_{\theta} / \mathcal{J}_{i}$ for all $i$. However, $C_{\delta} / \mathcal{I}$ is not isomorphic to $C_{\theta} / \mathcal{J}$ since $\operatorname{cl}\left(\bigcup S_{i}\right)$ is not homeomorphic to $\operatorname{cl}\left(\bigcup T_{i}\right)$. Notice that the conditions $\mathcal{I}_{i} \bigvee \mathcal{I}_{j}=C_{\delta}$ and $\mathcal{J}_{i} \bigvee \mathcal{J}_{i}=C_{\theta}$ are trivially satisfied.

The only way that the Chinese Remainder Theorem will work for a collection of ideals $\left\{\mathcal{I}_{i}\right\}$ indexed by some set $\Lambda$, is for the $S_{i}$ 's to be "discrete" (i.e. not to have any limit points). However, this is not possible in a compact space. Thus, one would not expect the Chinese Remainder Theorem to extend to infinitely many ideals.

When neither $X$ nor $Y$ is locally compact, one might try to "approximate" $K(X)$ and $K(Y)$ by sequences $\left[\alpha_{n} X, \beta X\right]$ and $\left[\gamma_{n} Y, \beta Y\right]$ where $\left\{\alpha_{n} X\right\}$ and $\left\{\gamma_{n} Y\right\}$ are decreasing sequences of compactifications in $K(X)$ and $K(Y)$ respectively. Then you could try to use Theorems 14 and 15 to get an isomorphism between $K(X)$ and $K(Y)$. However, this is not possible in general as the next example clearly shows.

Example. Let $X=\left\{(x, y) \in \mathbb{R}^{2}: 0 \leq x \leq 1,0 \leq y<1\right\} \cup\{(0,1)\}$ and $Y=$ $\left\{(x, y) \in \mathbb{R}^{2}: 0 \leq x \leq 1,0 \leq y<1\right\}$. Then $X$ is not locally compact while $Y$ is. Thus, $K(X)$ is not isomorphic to $K(Y)$.

Let $S_{n}=\operatorname{cl}_{\beta X}([1 / n, 1] \times[0,1)) \cap(\beta X \backslash X)$ and $T_{n}=\operatorname{cl}_{\beta Y}([1 / n, 1] \times[0,1)) \cap$ $(\beta Y \backslash Y)$. Let $\mathcal{I}_{n}=\left\{f:\left.f\right|_{S_{n}}=0\right\}$ and $\mathcal{J}_{n}=\left\{f:\left.f\right|_{T_{n}}=0\right\}$. There are compactifications $\alpha_{n} X$ and $\gamma_{n} Y$ so that $C_{\alpha_{n}}=\mathcal{I}_{n} \oplus 1$ and $C_{\gamma_{n}}=\mathcal{J}_{n} \oplus 1$. Then 
$\alpha_{n} X$ and $\gamma_{n} Y$ are decreasing sequences of compactifications and for each $n$, we know that $T_{n}$ is homeomorphic to $S_{n}$, so that $K\left(X, \alpha_{n}, \beta\right) \cong K\left(Y, \gamma_{n}, \beta\right)$.

\section{ACKNOWLEDGMENTS}

The author would like to thank Dr. George Cain, Dr. Tom Morley, and Dr. Bill Green for many useful conversations on this (and many other) topics. The author would also like to thank the referee for the comments which substantially improved the readability of the paper.

\section{REFERENCES}

[AB] J. M. Aarts and P. Van Emde Boas, Continua as Remainders in Compact Extensions, Nieuw Archief voor Wiskunde 3, XV (1967), 34-37. MR 35:4885

[Ch] Richard Chandler, Hausdorff Compactifications, Lecture notes in pure and applied mathematics 23, Marcel Dekker, New York, 1976. MR 58:24191

[F] P. A. Firby, Lattices and Compactifications I, II, and III. Proc. London Math Soc. 27 (1973), p. 22-68. MR 48:3001

[GJ] L. Gillman and M. Jerison, Rings of Continuous Functions, Graduate Texts in Mathematics 43, Springer-Verlag, New York, 1976. MR 53:11352

[MG1] K. D. Magill, Jr., A note on compactifications, Math. Zeitschr. 94 (1966), p. 322-325. MR 34:3530

[MG2] K. D. Magill, Jr., The lattice of compactifications of a locally compact space, Proc. London Math. Soc. 18 (1968), p. 231-244. MR 37:4783

[RAY] Marlon C. Rayburn, On Hausdorff Compactifications, Pac. J. Math. 44 (1973), 707-714. MR 47:5824

[RO] Joseph Rotman, An Introduction to the Theory of Groups, Wm. C. Brown Publishers, Dubuque, Iowa, 1988.

[PW] Jack R. Porter and R. Grant Woods, Extensions and Absolutes of Hausdorff Spaces, Springer-Verlag, New York, 1988. MR 89b:54003

[T] T. Thrivikraman, On the lattices of compactifications, J. London Math Soc. 4 (1972), p. 711-717. MR 45:5961

Applied Mathematics, University of Waterloo, Waterloo, Ontario, Canada N2L 3G1 E-mail address: mendivil@augusta.math.uwaterloo.ca

Current address: Department of Mathematics, Georgia Institute of Technology, Atlanta, Georgia $30332-0160$

E-mail address: mendivil@math.gatech.edu 